views of two senior scientists.

A delightful curiosity is the text of an address given by Einstein at Kyoto University on 14 December 1922. "How I Created the Theory of Relativity". The lecture was given in German and a Japanese physicist. J. Ishiwara, provided a translation. In 1923 Ishiwara published his Japanese text and in 1982 Physics Today printed an English translation by Yoshimasa A. Ono. Clearly, knowing that the lecture was to be translated, Einstein had formulated his ideas as concisely and simply as possible, but even so it is remarkable to see how well the text has survived two consecutive translations. There are several other articles in which the story of an important discovery or invention is told by people who were not necessarily the only originators but who did play an important part - Frisch and Wheeler on the discovery of fission is a good example, as is the history of the cyclotron told by Livingston and McMillan.

Also included are several biographical essays. Most of these are written by authors who were well-acquainted with their subjects, as their students or collaborators, which makes the stories lively and readable - here, Oliphant's "The Two Ernests" (Rutherford and Lawrence) is especially illuminating. Quite different again are the scholarly papers on specific aspects of the history of physics, for example Martin Klein's thorough study "Thermodynamics and Quanta in Planck's Work". Klein shows how Planck, a conservative rather than a revolutionary, found it hard to reconcile himself to his own innovation, but finally arrived at the conclusion "that the quantum of action played a far more significant part in phy. sics than I had originally been inclined to suspect".

It is understandable that the contributions on institutional history deal almost exclusively with American establishments, and the same is true, though to a lesser extent, of the articles grouped under the heading "Social Context". But this does not mean that they are of no interest to non-Americans, and in many cases the problems discussed are international in nature. Of particular interest here is the article by Vera Kistiakowski with the provocative title "Women in Physics: Unnecessary, Injurious and Out of Place?".

Few people will read this book from cover to cover - the editors even warn against trying to do this. But all physicists and many non-physicists will enjoy leafing through it, will admire the many excellent illustrations, and will sooner or later start reading in earnest.

$\overline{\text { Hendrik B.G. Casimir, De Zegge } 75591 \text { TT }}$ Heeze, The Netherlands, studied theoretical physics at Leiden, Copenhagen and Zürich during the 1920s and 1930s and later became joint director of research at the Philips Company, Eindhoven. His autobiography, Haphazard Reality: Half a Century of Science, was published by Harper \& Row in 1983.

\section{In the realm of the cosmocrats}

\section{Edward Harrison}

The Cosmological Distance Ladder: Distance and Time in the Universe. By Michael Rowan-Robinson. W.H. Freeman: 1985. Pp.353. \$35.95, £19.95.

COMTE de Buffon once said that the forms of life seem more like chain mail than a Great Chain of Being. Similarly the measure of the universe seems more like a network of ladders than a single cosmological distance ladder. (An irreverent person might say more like a game of snakes and ladders.)

The scale of the universe has been increased several times since Hubble wrote The Realm of the Nebulae in 1936. The Hubble term $H$ in the velocity-distance law (velocity $=H \times$ distance) has decreased in value by an order of magnitude and now stands at either 100 or $50 \mathrm{~km} \mathrm{~s}^{-1} \mathrm{Mpc}^{-1}$. The de Vaucouleurs school champions the higher value and the Sandage-Tammann school the lower value. The reciprocal of $H$ is about fifteen thousand million years and serves as a rough measure of the age of the universe.

Rowan-Robinson unifies his splendid and comprehensive survey of the universe - planets, stars, star clusters, galaxies, galaxy clusters, superclusters - with the theme of the cosmological distance ladder. In the preface he writes that, at a conference in 1976 ,
Gerard de Vaucouleurs on the one hand, and Allan Sandage and Gustav Tammann on the other, arrived at estimates of the size of the universe, as measured by the Hubble constant, differing from each other by a factor or two. Moreover, when I asked the protagonists what was the range outside which they could not imagine the Hubble constant lying, these ranges did not even overlap. ... It was to understand this disagreement that $I$ set out to write this book.

Using numerous figures, tables and appendices that display a wealth of data, the author builds an array of distance indicators and presents a difficult subject in a methodical manner. Skilfully he adjusts, deftly weighs, and adroitly averages divergent results.

The first rung on the ladder is the distance between the Sun and the Earth. Then comes the distance to the Hyades, the nearest open star cluster, determined by parallactic and moving cluster methods. The size of this preliminary and important step, now estimated to be 150 light-years, has grown 30 per cent since 1940.

We measure in Hyades-units the distance to other open clusters, applying various methods including evolutionary curve fitting. Then, with allowance for absorption of light by interstellar dust, we use Cepheids and RR Lyrae variable stars as yardsticks to gauge the distances of globular clusters in our Galaxy and of nearby galaxies in the Local Group (rival schools going different ways and making different allowances for extinction of light). Novae, supernovae and HII regions, adjusted for extinction in the observed galaxy, serve as intergalactic
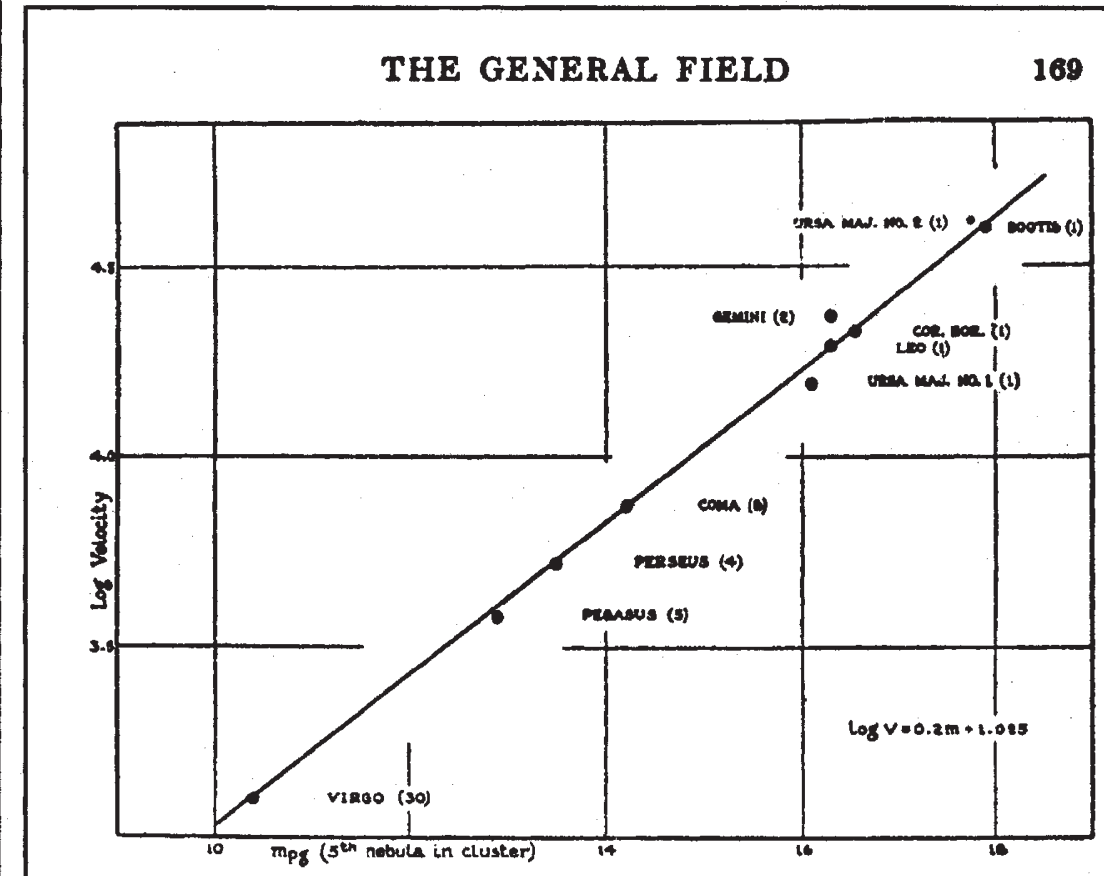

Fra. 14. Velocity-Distance Relation for Clusters.

169

(Taken from E. Hubble's The Realm of the Nebulae.) 
indicators of distance; here the methods become less reliable and the uncertainties increase yet further. Supergiant galaxies then act as stepping stones to remoter regions.

It began with the Greeks. RowanRobinson gives them a page or two and then jumps, as one expects in an astronomer's history of astronomy, from Aristotle to Copernicus. The Epicurean system (an infinite atomic universe) and Stoic system (an island cosmos surrounded by an infinite extracosmic void) receive scant attention, although both dominated the intellectual climate of the ancient world. The great historical drama of the mediaeval revival of the Aristotelian system, the eclipse of this system in the late Middle Ages by a renascent Stoic system, the rebirth of the Epicurean system, and the eventual overthrow of the Stoic system in the Great Debate of the 1920 s also receives little recognition. We are told that in the seventeenth century Cassini measured the Sun-Earth distance to within 10 per cent of the modern value, but not that Newton, by using James Gregory's photometric method of comparing the outer planets with the brightest stars, determined with astounding accuracy (within a factor of two for Sirius) the distance of the nearest stars.

In a chapter entitled "The Cosmological Models", which is devoted to theoretical topics, the author discusses dynamic models, redshifts, proper and luminosity distances, the early universe, and the motion of the Solar System relative to the microwave background and to the Local Supercluster. Perhaps more such analysis in the other chapters would have helped the reader to surmount the mountains of data to be found in them.

The axioms or irreducible assumptions of modern cosmology rarely receive adequate attention, and the book would have benefited from some discussion of these hidden assumptions. The Hubble (and deceleration) term tell us how the universe expands. But how exactly do we need to know its value? Theorists first postulated the velocity-distance law, which applies at any instant throughout a homogeneous expanding universe. But when we look out in space we also look back in time, and homogeneity (unlike isotropy) cannot be directly seen. Observations made on the backward light cone are adjusted for evolutionary effects and mapped in a hypothetical homogeneous space. At best we verify that redshifts and estimated distances are consistent within the theoretical frame of a geometric and dynamic model that is itself unverified. Perhaps our models are too simple and we do not know enough cosmology to make an exact value of the Hubble term meaningful?

Edward Harrison is Professor of Astronomy in the Department of Physics and Astronomy. University of Massachusetts, Amherst. Massachusetts 01003, USA.

\section{Halley's comet in print}

\section{David W Hughes}

ON 16 October 1982 Halley's comet was picked up using a charge-coupled device at the prime focus of the $5.1 \mathrm{~m}$ Hale telescope on Mount Palomar. It has been monitored ever since. As it approaches the Sun it brightens and will become just visible to the naked eye between December 1985 and April 1986. But there are two problems for observers. On 9 February 1986 the comet is at its closest to the Sun and for a six-week period around then is in too bright a region of sky to be easily visible. Also, between December and April it sweeps from the northern to the southern sky, requiring the northern observer to reciprocate by moving to the southern hemisphere. Unfortunately planet Earth does not get very close to the comet this time, so we are not going to be overawed by startling searchlight-like beams radiating up from the twilight horizon. In fact the mere act of catching sight of the comet will require some effort.

Halley's return to the inner Solar System has been greeted by a whirlwind of books, and thus a rather rare state of affairs as far as the reviewer is concerned. Most of them in fact say much the same things, use the same illustrations and are aimed at a like audience. Most are written by non-specialists who, for simplicity, have consulted only secondary sources of information and have consequently made many mistakes. Reviewing them is akin to essay-marking. To help the prospective buyer the books are divided into categories, with a star rating for each according to whether it is thought to be excellent, good, indifferent or poor. The five-star rating is reserved for those rare books in the pile that will "last", to be consulted around AD 2061 when Halley's comet returns once more.

The categories are as follows: (i) Books specifically designed to help the reader see the comet (the message being that you have a-once-in-a-lifetime chance so make the most of it); (ii) guides for teachers, that is books specifically designed for the organizers of classes; (iii) specialist books; and (iv) general books.

Halley's Comet by Francis Reddy leads the first category. It abounds with good advice: "If you want to see the comet you had better prepare for it, familiarize yourself with the constellations through which Halley moves, get away from cities, use your binoculars and travel south". Twenty maps are provided, for three Earth latitudes $\left(55^{\circ} \mathrm{N}, 40^{\circ} \mathrm{N}\right.$ and $\left.35^{\circ} \mathrm{S}\right)$. These not only show the comet and its tail against the starry background but also include (where appropriate) the Moon, Mars and Jupiter. A superb three-dimensional model of the orbits of the comet and Earth is an additional bonus.

Such models are a great help when it comes to visualizing the movement of the comet with respect to the Sun and Earth. and Perihelion Scientific have produced one, Halley's Comet Survival Kit, which is $85 \mathrm{~cm}$ across. Another accompanying model enables you to calculate the altitude and azimuth of the comet throughout the November 1985-May 1986 period. Both are great fun to assemble and use.

If you are happier with a planisphere, David Chandler has designed a set of three (for northern latitudes $26^{\circ}, 35^{\circ}$ and $44^{\circ}$ ). These show the comet's path through the constellations and it is simplicity itself to calculate when and where it can be seen. Unfortunately there are no hints as to its brightness.

Halley's Comet Finder by Ben Mayer is aimed more at the astronomically initiated and gives a detailed review of the comet's journey across the sky. The section on astrophotography in this book is most useful.

For the complete beginner the editors of Sky and Telescope have produced $\mathrm{Mr}$. Halley's Comet (I've no idea why they refer to a man who was an eminent professor and had two doctorates as "Mr."). This is an excellent and inexpensive guide mar. red only by the sparsity of stars on the sky map. The fact that a pair of binoculars has only a limited field of view is rightly stressed, but the difficulty of finding the comet and recognizing stars in the field is somewhat overlooked. The guide seems to be designed in the hope that the comet would be much brighter.

Moving into the classroom, both Comet Halley Returns and Halley's Comet Activities Manual get good marks and will provide science teachers and their scholars with hours of activity understanding comets, modelling their orbits, recording observations, computing examples of Keplerian motion, introducing the life and times of Edmond Halley and trying to appreciate his achievement. Halley's Comet: Teacher's Notes comes with posters and is biased towards young $(9-14)$ or innumerate pupils (and supposedly innumerate teachers too!). It approaches cometary science from a sociological and refreshingly critical standpoint - I'm still considering "the possibility that women astronomers do not appear in astronomy books because those books are generally written by men", one of the topics suggested for classroom discussion. Mary Ashby, the author, has a clear grasp of what children find interesting and what they find difficult to understand, and has planned a series of exercises for explaining difficult concepts.

Nine books fall into the specialist category, two of which are bibliographies. Ruth S. Freitag lists 3,289 references to both popular and scientific cometary literature. The list is alphabetical by author and each 